\title{
R. GOULET (éd.), Dictionnaire des philosophes antiques, VI. De Sabinillus à Tyrsénos; VII. D'Ulpien à Zoticus
}

Paris, CNRS éditions, 2016, 1330 p., ISBN 978-2-271-08989-2 et 2018, 1472 p., ISBN 978-2-271-09024-9.

\section{Pierre Pontier}

\section{(2) OpenEdition}

1 Journals

\section{Édition électronique}

URL : https://journals.openedition.org/philosant/1833

DOI : $10.4000 /$ philosant. 1833

ISSN : 2648-2789

Éditeur

Éditions Vrin

\section{Édition imprimée}

Date de publication : 31 octobre 2019

Pagination : 213-214

ISBN : 978-2-7574-2534-3

ISSN : 1634-4561

\section{Référence électronique}

Pierre Pontier, «R. goulet (éd.), Dictionnaire des philosophes antiques, VI. De Sabinillus à Tyrsénos ; VII. D'Ulpien à Zoticus », Philosophie antique [En ligne], 19 | 2019, mis en ligne le 20 janvier 2019, consulté le 02 décembre 2022. URL : http://journals.openedition.org/philosant/1833 ; DOI : https://doi.org/ 10.4000/philosant.1833

\section{(c) $(1)$}

Creative Commons - Attribution - Pas d'Utilisation Commerciale - Pas de Modification 4.0 International - CC BY-NC-ND 4.0

https://creativecommons.org/licenses/by-nc-nd/4.0/ 
dont ils sont exclus signifie. Néanmoins, ces deux remarques n'entament pas la valeur d'ensemble du livre, dont tout l'intérêt est de mettre à jour des mécanismes et des enjeux significatifs dans la construction d'une notion omniprésente depuis l'Antiquité dans la pensée et la pratique politiques occidentales.

Étienne HeLmer

Université de Porto Rico - etiennehelmer@hotmail.fr

R. Goulet (éd.), Dictionnaire des philosophes antiques, VI, Paris, CNRS éditions, 2016, 1330 p., ISBN 978-2-271-08989-2 ; VII, Paris, CNRS éditions, 2018, 1472 p., ISBN 978-2-271-09024-9.

Avec la publication des tomes VI (2016) et VII (2018) du Dictionnaire desphilosophes antiques, l'entreprise «biobibliographique » colossale menée par Richard Goulet touche à sa fin. On trouve dans ces deux tomes la même rigueur éditoriale et recherche d'exhaustivité que dans les cinq premiers et l'on reste admiratif et reconnaissant devant le travail de coordination, de rédaction et de relecture du maittre d'œuvre. Ce dernier est aussi le principal contributeur en nombre de notices du dictionnaire, étant l'auteur de 135 notices sur les 373 que compte le volumineux tome VI (1330 pages) qui couvre les lettres $S$ et T. Le dictionnaire donne parfois l'impression d'être un incessant « work in progress », comme en témoignent par exemple les « compléments » introduits p. 1273 à 1276 aux notices « Simplicius », « Théano » et «Timarès », ou la double rubrique « iconographie » (p. 180 sq. et 201 sq.) dans la notice consacrée à Sénèque, subtilités formelles qui doivent inciter le lecteur à être très scrupuleux au cours de sa consultation. L'éditeur l'y aide toujours par les nombreux index et renvois internes. Il a également dû composer avec la spécialisation toujours plus grande des champs de recherche : la notice «Socrate » est ainsi répartie entre quatre auteurs différents; sur les cinquante-six pages de la notice, dix-neuf sont consacrées à Socrate lui-même jusqu’à la période hellénistique, trente à la réception de Socrate dans le néoplatonisme et dans la tradition arabe, sept enfin traitent de l'iconographie. La lecture successive de ces quatre parties témoigne de l'importance prise dans nos disciplines par les études de réception et par l'iconographie, mais aussi de conceptions sensiblement différentes du dictionnaire en fonction des rédacteurs, du guide qui oriente avec rigueur, plus proche de la clavis (M. Narcy, p. 415), au guide qui expose (D. Alexandra Layne). Peu importe au demeurant, puisque le guide est bon.

Rien ne laisse présager un tome VIII, puisqu'un Supplément a été publié en 2003 et que le tome VII comprend une rubrique « Compléments » qui intègre de nombreuses notices qui complètent les tomes antérieurs, les lettres $\mathrm{U}$ à $\mathrm{Z}$ n'occupant que le tiers du volume. Parmi les notices, on relèvera, de façon tout à fait subjective, celle consacrée à Varron, qui réévalue l'importance de son œuvre philosophique (pour l'essentiel perdue par la tradition, voir par exemple les pages consacrées à la «théologie tripartite » de Varron), alors qu'on ne retient d'ordinaire que le grammairien ou l'auteur des Res rusticae (p. 94-133), l'importante synthèse de Louis-André Dorion sur Xénophon, définitivement réintroduit dans le cercle des philosophes antiques, celle consacrée à Virgile, apparemment incongrue mais justifiée par l'éducation reçue, les cercles épicuriens fréquentés par le poète, et l'influence sur l'œuvre poétique de l'enseignement mathématique reçu. La notice vertigineuse consacrée à Pythagore, tradition syriaque et arabe incluse, occupe à elle seule 200 pages, auxquelles on doit ajouter 200 autres pages qui traitent de l'histoire de l'école pythagoricienne et de la réception du pythagorisme, 
bizarrement placées pour des raisons éditoriales en fin de volume dans l'annexe II (p. 10251174). On devine que le tout n'a pas dû être aisé à rédiger, tant la « figure énigmatique et élusive » de Pythagore échappe au projet concret d'une notice de dictionnaire ( $c f$. le résumé méthodologique sur la « question pythagoricienne », p. 694); elle a en outre suscité un nombre de publications inversement proportionnel au nombre de documents avérés conservés - même le dossier iconographique s'avère rempli d'incertitudes et laisse « Pythagore enveloppé à jamais dans l'aura idéalisante de la légende » (p. 760-761). L'Annexe I comprend quatre courtes notices consacrées à divers lieux d'enseignement philosophique (le Lycée, la Stoa Poikilè, le « Jardin » et Apamée). Suit enfin un étonnant « epimetrum », qui propose un ensemble de statistiques commentées sur les philosophes de l'Antiquité à partir de l'« échantillon » que constitue le $D P h A$. Il permet d'esquisser quelques tendances (prudentes) sur ce qu'est un philosophe en fonction des périodes et des écoles. Selon Diderot, un epimetrum est « la partie de la cargaison totale d'un vaisseau, qu'on accordait aux pilotes, et dont ils pouvaient disposer à leur profit », et, si l'on file la métaphore, l'un des tableaux le plus éclairant et nostalgique est sans doute celui qui montre comment le vaisseau philosophique a rapidement sombré entre le $\mathrm{v}^{\mathrm{e}}$ et le vi ${ }^{\mathrm{e}}$ siècles de notre ère (graphique 1, p. 1196). Le reste du volume VII comprend l'index indispensable des noms propres, boussole ultime à laquelle on se reportera en priorité pour se repérer dans le $D P h A$, ainsi qu'une série d'index complémentaires. En somme, nous disposons désormais de l'outil complet pour nos recherches, et l'on peut dire en le consultant ce qu'écrivait le péripatéticien Serenus sur les murs du tombeau de Ramsès

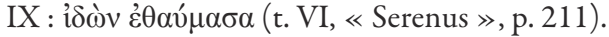

Pierre Pontier Sorbonne Université 